\title{
Children's Mouthwash; Commercial Product or Oral Health Guarantor
}

\author{
Karimi M, D.M.D* \\ Sepideh Dental Clinic, Department of Pediatric Dentistry, Iran \\ *Corresponding author: Karimi M, Sepideh Dental Clinic, Department of Pediatric Dentistry, Iran
}

\section{Opinion}

Nowadays, when we enter a pharmacy, we come across a variety of healthcare products that come to the market every day. Types of masks, gels, detergents and etc., which may look identical in name and application but claim to be totally different and each has its own user extent. Some of these products are commercial in nature, and some are not so important in maintaining health, and in fact, one's health is not dependent on them. Perhaps that's why each of us never consumed some of these health and beauty products, and we do not even know them at all. If the product does not directly interact with our health, we do not need to carefully look at the need to how to consume them and do not curious about them, but when it comes to products that claim to protect our child's health, the matter becomes more quite critical and worrying. Mouthwash is one of these products which are found at all pharmacies. So many oral health officials have been able to highlight the need to use mouthwashes among people. With the training and information provided in this area, people should use mouthwashes in addition to toothpaste and dental floss. But what is the child's mouthwash, and do the children really need them to use? Do these products guarantee the health of their teeth or is it more a commercial product to fill the pockets of some of the companies?

Mouthwashes have different types. Some have pharmaceutical uses and should be used in certain cases only with physicians' prescriptions. Mouthwashes that are on the market for the public use contains fluoride which prevents tooth decay. Many pharmaceutical companies have produced and marketed mouthwashes for children to facilitate the use of mouthwashes for children. These mouthwashes have been manufactured considering the least risk of swallowing fluoride. The proper taste of these mouthwashes is a feature that encourages children to use them. Mouthwash is one of the complementary methods of oral home care. These mouthwashes provide oral and dental care along with tooth brushing and dental floss, but they should never be replaced by each other. In other words, application of none of them alone has the significant effect. Before planning to buy the product, if parents tend to use pediatric mouthwashes, they should have a consultation with the pediatric dentist so that the dentist can select the better of the most appropriate one and the least harmful mouthwash. However, they should be used according to the instructions; these instructions vary from product to product and depending on the content and concentration of fluoride, the application may be different.

In other words, it should be noted that some types of mouthwashes are highly recommended by most dentists in the routine oral care program. This is due to the ease and speed of its use, and its effectiveness. In general, along with toothbrushes and dental flosses, many types of mouthwashes are also produced, each of which has its own interests in the beauty and health of teeth. These mouthwashes can be prescribed by the dentist or can be purchased OTC from the pharmacy. Nonetheless, alcohol-free mouthwash is a product that depends on the individual's needs. The choice of the mouthwash that meets personal needs is very important. Some mouthwashes on the market contain alcohol, in particular, Ethanol, which can cause burning sensation, unpleasant taste, and dryness in the mouth. It is not recommended for children at all because the burning and spicy tastes force the child to stop using the kid's mouthwash for future use. Consequently, if the parents intend to choose an appropriate mouthwash, first they should not choose any kinds of mouthwashes, and secondly, it is advisable to have a consultation with the pediatric dentist so that they don't become bewilderment when choosing the proper mouthwash. To finish this point of view, for some time, fluoridecontaining mouthwash has been commonly used in children. But, in my opinion, some kids do not need to use them at all. That is, if 
fluoride is adequately contained in fluoride-containing toothpaste, in drinking water, or even the consumption of foods such as seafood or tea which contains this material, then, there is usually no need for fluoride mouthwashes for children.

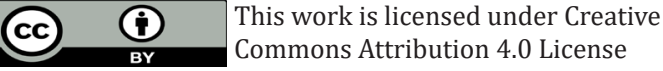

To Submit Your Article Click Here: $\quad$ Submit Article

DOI: 10.32474/MADOHC.2018.03.000164

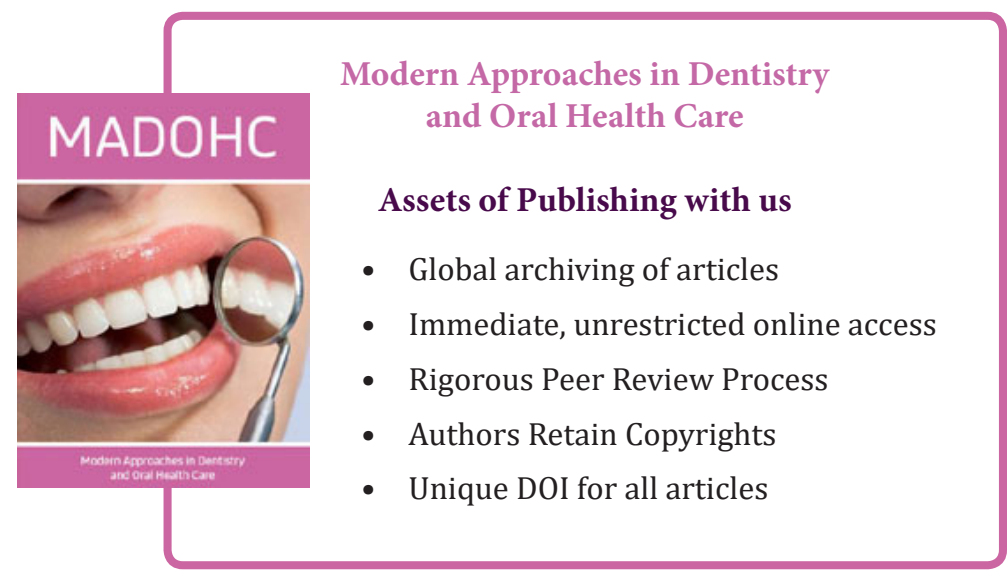

